\title{
Rifabutin Suppresses Inducible Clarithromycin Resistance in Mycobacterium abscessus by Blocking Induction of whiB7 and erm41
}

\author{
Dinah Binte Aziz ${ }^{1}$, Mei Lin Go ${ }^{1}$ and Thomas Dick ${ }^{2,3, *}$ (1) \\ 1 Department of Pharmacy, Faculty of Science, National University of Singapore, 18 Science Drive 4, Singapore \\ 117543, Singapore; a0084914@u.nus.edu (D.B.A.); meilin.go@nus.edu.sg (M.L.G.) \\ 2 Department of Medical Sciences, Hackensack Meridian School of Medicine at Seton Hall University, \\ 340 Kingsland Street, Nutley, NJ 07110, USA \\ 3 Center for Discovery and Innovation, Hackensack Meridian Health, 340 Kingsland Street Building 102, \\ Nutley, NJ 07110, USA \\ * Correspondence: thomas.dick@hmh-cdi.org
}

Received: 21 December 2019; Accepted: 6 February 2020; Published: 10 February 2020

\begin{abstract}
Clarithromycin (CLR) is the corner stone in regimens for the treatment of lung disease caused by Mycobacterium abscessus (Mab). However, many strains harbor the CLR-inducible CLR resistance gene erm 41 , encoding a ribosome methylase. Induction of erm 41 is mediated by the transcription factor whiB7. We hypothesized that an inhibitor of RNA synthesis should be able to block the whiB7-erm 41 induction response to CLR exposure and thus suppress CLR resistance. Recently, we discovered that the rifampicin analog rifabutin (RFB) shows attractive potency against $M a b$. To determine whether RFB-CLR combinations are synergistic, a checkerboard analysis against a collection of erm41 positive and negative Mab strains was carried out. This revealed synergy of the two drugs for erm 41 positive but not for erm 41 negative strains. To determine whether RFB's potentiation effect was due to inhibition of the transcriptional induction of the whiB7-erm 41 resistance system, we measured the effect of CLR alone and in combination with RFB on whiB7 and erm 41 mRNA levels. CLR alone strongly induced whiB 7 and erm 41 expression as expected. The synergistic, growth-inhibiting combination of RFB with CLR blocked induction of both genes. These results suggest that RFB suppresses inducible CLR resistance by preventing induction of whiB7 and erm41 expression.
\end{abstract}

Keywords: synergy; non-tuberculous mycobacteria; NTM; rifabutin; clarithromycin; whiB7; erm41

\section{Introduction}

Mycobacterium abscessus (Mab) causes difficult-to-cure lung disease. Current multidrug-regimens typically include clarithromycin (CLR) (or its analog azithromycin), amikacin, imipenem and tigecycline [1-3]. Rifampicin, a key drug used for the treatment of Mycobacterium tuberculosis, shows poor activity against Mab and is not used clinically. CLR is the cornerstone of Mab treatment and resistance against CLR is associated with poor outcomes [4,5]. Many Mab strains are intrinsically resistant against CLR. Intrinsic resistance is mediated by Erm41 [6]. Erm41 is a methylase that modifies the binding site of CLR on the $23 S$ ribosomal RNA. Intrinsic CLR resistance is inducible. Exposure to subinhibitory concentrations of CLR induces transcription of whiB7 encoding a transcriptional activator, which in turn induces transcription of erm41 [6-9].

Mab represents a complex of three subspecies, Mab subsp. abscessus, Mab subsp. bolletii and Mab subsp. massiliense [10]. Interestingly, all Mab strains carry erm 41 sequences, but only a subset harbors functional erm 41 alleles, i.e., alleles that confer phenotypic CLR resistance ('erm 41 positive' strains). 
The others ('erm 41 negative') are CLR susceptible strains that carry deletions or a T to C polymorphism at position 28 of the coding sequence of the gene, resulting in loss of Erm41 activity [11-14].

New drugs that address CLR resistant Mab infections, are urgently needed [15]. In a repurposing project, we recently screened a library of approved drugs and found attractive in vitro activity in the rifampicin analog rifabutin (RFB) [16]. Subsequently, we demonstrated activity of RFB in a mouse model of Mab infection [17], and a first report on the beneficial use of the drug in patients suffering from disseminated Mab disease was published [5]. This suggests that RFB is a repurposing candidate for the treatment of Mab lung infections. Based on the finding that inducible CLR resistance appears to be under transcriptional control and that exposure to CLR increases the mRNA levels of both whiB7 and erm41, we hypothesized that treatment of Mab cultures with RFB should suppress inducible resistance. The RNA polymerase inhibitor should block transcriptional induction of the whiB7-erm 41 resistance system and thus hold the genotypically CLR resistant Mab in a phenotypically CLR susceptible state. This hypothesis is supported by recent synergy studies that suggest that RFB can potentiate the activity of CLR $[18,19]$. If confirmed, this would suggest that intrinsically CLR-resistant Mab disease due to erm 41 could become treatable with CLR by adding RFB. Here, we tested this hypothesis by determining the effect of RFB on CLR activity in erm41 positive and negative strains in vitro, and by determining the effect of RFB on transcript levels of whiB7 and erm41.

\section{Results}

The RFB-CLR combination is synergistic for erm 41 positive and indifferent for erm 41 negative M. abscessus. If RFB suppresses inducible CLR resistance, RFB should potentiate the effect of CLR. This potentiation effect should result in synergy of the two drugs. The synergistic effect of CLR-RFB should be specific for erm 41 positive Mab strains and should not be observed for erm 41 negative strains. To test these predictions, we carried out a checkerboard assay to determine drug-drug potency interactions. The assay was carried out with a collection of reference strains and clinical isolates covering all three subspecies and containing both erm 41 positive and negative strains. The CLR-RFB combination was indeed synergistic against all erm41 positive strains (Table 1). This suggests that RFB potentiates the effect of CLR in this genetic background, as expected if RFB suppresses the expression of CLR resistance and thus increases the susceptibility to the antibiotic. Furthermore, as predicted, the RFB-CLR combination was indifferent to all erm 41 negative Mab strains, demonstrating that RFB required the presence of inducible erm 41 to potentiate the activity of CLR. Thus, RFB potentiates CLR in an erm41 dependent manner. It is interesting to note that the outcomes of the drug-drug interaction studies were solely dependent on the erm 41 status (type of erm 41 sequevar) and not influenced by the type of subspecies. This is illustrated by the difference in outcome between the two Mab subspec. abscessus isolates Bamboo (erm41 negative) and Mab ATCC19977 (erm41 positive), and the two Mab subspecies bolletii isolates M506 (erm41 negative) and CCUG 50184-T (erm41 positive). Independent of the subspecies, RFB-CLR synergy was observed for erm 41 positive members of the respective subspecies (Table 1). In summary, the checkerboard analyses showed that the RFB-CLR combination is synergistic for erm 41 positive and indifferent for erm 41 negative Mab strains. This shows that the potentiation effect of RFB on CLR is erm 41 dependent. Thus, co-treatment of Mab strains displaying inducible CLR resistance with RFB appears to restore activity of CLR. 
Table 1. Checkerboard assay results for the combination of clarithromycin and rifabutin against three reference strains and a collection of clinical isolates of M. abscessus.

\begin{tabular}{|c|c|c|c|c|c|c|}
\hline Isolate Code & $\begin{array}{l}\text { M. Abscessus } \\
\text { Subspecies }\end{array}$ & $\begin{array}{l}\text { Erm41 } \\
\text { Sequevar }\end{array}$ & Erm41 Status & $\begin{array}{l}\text { CLR } \\
\text { Susceptibility }\end{array}$ & CLR + RFB & FICI \\
\hline ATCC 19977 & abscessus & $\mathrm{T} 28$ & Functional & Resistant & $S$ & 0.26 \\
\hline Bamboo & abscessus & $\mathrm{C} 28$ & Non-functional & Sensitive & I & 0.56 \\
\hline M9 & abscessus & $\mathrm{T} 28$ & Functional & Resistant & $S$ & 0.39 \\
\hline M199 & abscessus & $\mathrm{T} 28$ & Functional & Resistant & $S$ & 0.39 \\
\hline M337 & abscessus & $\mathrm{T} 28$ & Functional & Resistant & $S$ & 0.39 \\
\hline M421 & abscessus & $\mathrm{T} 28$ & Functional & Resistant & $S$ & 0.49 \\
\hline M422 & abscessus & $\mathrm{T} 28$ & Functional & Resistant & $S$ & 0.39 \\
\hline $\begin{array}{l}\text { CCUG } \\
50184-T\end{array}$ & bolletii & $\mathrm{T} 28$ & Functional & Resistant & S & 0.32 \\
\hline M232 & bolletii & $\mathrm{T} 28$ & Functional & Resistant & $S$ & 0.21 \\
\hline M506 & bolletii & $\mathrm{C} 28$ & Non-functional & Sensitive & I & 0.77 \\
\hline $\begin{array}{l}\text { CCUG } \\
48898-\mathrm{T}\end{array}$ & massiliense & deletion & Non-functional & Sensitive & I & 0.77 \\
\hline M111 & massiliense & deletion & Non-functional & Sensitive & I & 0.78 \\
\hline M353 & massiliense & deletion & Non-functional & Sensitive & I & 0.65 \\
\hline M357 & massiliense & deletion & Non-functional & Sensitive & I & 1.05 \\
\hline M414 & massiliense & deletion & Non-functional & Sensitive & I & 0.86 \\
\hline M444 & massiliense & deletion & Non-functional & Sensitive & I & 0.85 \\
\hline M505 & massiliense & deletion & Non-functional & Sensitive & I & 1.17 \\
\hline
\end{tabular}

Fractional inhibitory concentration index (FICI) values are shown as a measure of synergy. Synergy (S) is defined as $\mathrm{FICI} \leq 0.5$, indifference (I) is defined as $0.5<\mathrm{FICI} \leq 4$, and antagonism $(\mathrm{A})$ is defined as FICI $>4$. erm 41 status (functional $=e r m 41$ positive; non-functional $=e r m 41$ negative) and phenotypic CLR susceptibility of the strains were described previously [16].

Treatment of erm41 positive M. abscessus with RFB-CLR combination at synergistic, growth inhibiting concentrations suppresses induction of $w h i B 7$ and erm 41 expression. Treatment of erm 41 positive Mab with CLR induces transcription of whiB7 and erm41 [6,7]. The checkerboard analysis revealed that growth of the erm 41 positive Mab ATCC19977 is inhibited by a combination of CLR and RFB at 0.78 and $0.39 \mu \mathrm{M}$, respectively (Table 1 and Table S1). This compares to the MIC of the single drugs of $5 \mu \mathrm{M}$ (CLR) and $3 \mu \mathrm{M}$ (RFB). If this synergistic effect of the CLR-RFB combination is due to blocking induction of transcription of whiB7 and erm41, the mRNA levels of the two genes should not increase in cultures treated with the synergistic, growth inhibiting CLR-RFB combinations. To determine the effect of drug treatment on the mRNA levels of the two genes, cultures were treated for $30 \mathrm{~min}$ and quantitative reverse transcription PCR (qRT-PCR) was carried out. First, we confirmed that CLR alone at MIC $(5 \mu \mathrm{M})$ or a subinhibitory concentration of $0.78 \mu \mathrm{M}$ causes an increase of whiB7 and erm 41 mRNA levels as reported previously (Figure 1). Then we determined the effect of the synergistic, growth inhibitory RFB-CLR combination. Adding $0.39 \mu \mathrm{M}$ RFB to $0.78 \mu \mathrm{M}$ CLR suppressed the increase of both whiB7 and erm 41 transcript levels (Figure 1). Taken together, these results confirm that CLR treatment alone induces transcription of whiB7 and erm 41 and show that the addition of RFB blocks this induction. These results suggest that the observed potentiation effect of RFB on CLR is due to the inhibition of induction of the whiB7-erm41 resistance system. 


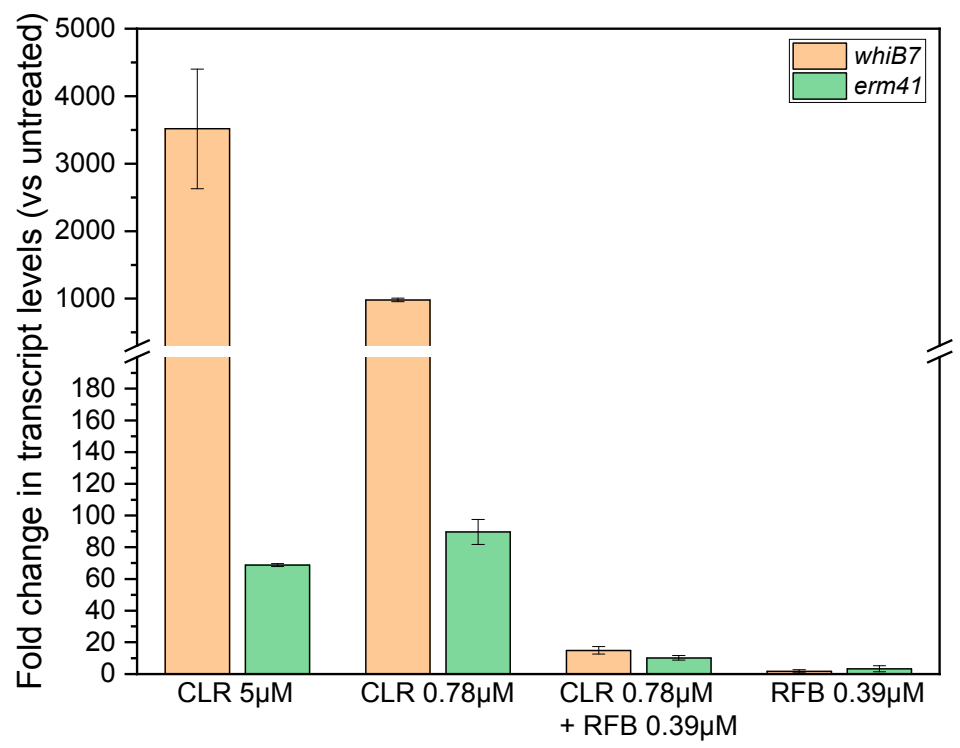

Figure 1. Effect of treatment of M. abscessus with clarithromycin and/or rifabutin on whiB7 and erm41 mRNA levels. M. abscessus ATCC19977 cultures were exposed for $30 \mathrm{~min}$ to clarithromycin (CLR), rifabutin (RFB) or a combination of both antibiotics and relative transcript levels were determined. Fold changes compared to untreated cultures are shown. CLR $5 \mu \mathrm{M}$ : MIC of CLR. CLR $0.78 \mu \mathrm{M}$ : subinhibitory CLR concentration used in the synergistic combination. CLR $0.78 \mu \mathrm{M}+\mathrm{RFB} 0.39 \mu \mathrm{M}$ : synergistic, growth inhibitory combination. RFB $0.39 \mu \mathrm{M}$ : subinhibitory RFB concentration used in the synergistic combination. Transcript levels were measured by qRT-PCR and normalized to rps $A$ as described [6]. Shown are mean values with standard deviations from two independent experiments. $0.78 \mu \mathrm{M}$ CLR corresponds to $1 \mathrm{mg} / \mathrm{L} ; 0.39 \mu \mathrm{M}$ RFB corresponds to $0.46 \mathrm{mg} / \mathrm{L}$.

\section{Discussion}

Repurposing and repositioning of antibacterials used in the clinic is an attractive alternative to de novo drug discovery [15]. To identify clinically used medications active against $M a b$, we recently screened a library of approved drugs and identified the rifamycin RFB to be active against Mab in vitro [16]. This was surprising because rifampicin, the corner stone drug for $M$. tuberculosis treatment, shows poor activity against $M a b$ and is therefore not used clinically. The reason for the difference in potency of the two rifamycins against $M a b$ remains to be determined but appears to involve bacterial metabolism $[20,21]$. Thus, RFB, being an approved oral drug, should be considered for repurposing as an add-on for the treatment of Mab lung disease. This notion is supported by our recent finding, that RFB, in contrast to rifampicin, also showed efficacy in vivo in a mouse model for Mab infection [17]. The first report suggesting that RFB may be beneficial for the treatment of patients suffering from disseminated Mab disease is encouraging [5].

CLR is a key component in current treatments of Mab lung disease. However inducible CLR resistance conferred by the whiB7-erm 41 resistance system is widespread among clinical isolates [11,12]. The induction of whiB7-erm 41 mediated CLR resistance appears to be regulated at the transcriptional level $[6,7,22,23]$. Thus, we asked here whether the RNA synthesis inhibitor RFB may suppress inducible CLR resistance. We show that RFB indeed potentiated the activity of CLR in erm41 positive strains (Table 1). This potentiation effect of RFB on CLR correlated with a suppression of the transcriptional induction of the whiB7 and erm 41 genes (Figure 1). These results show that RFB suppresses phenotypic expression of CLR resistance, i.e., RFB treatment appears to keep Mab in a CLR susceptible state. Furthermore, our results provide a mechanistic explanation for this effect, namely that RFB suppresses transcriptional activation of whiB7-erm41.

The presented work may have clinical implications. It suggests that RFB may not only be useful as an add-on to the regimens used for the treatment of Mab lung disease in general as suggested 
earlier [16], but may be particularly useful for disease caused by erm41 positive Mab as the rifamycin appears to restore CLR susceptibility of the genotypically resistant bacteria (Ganapathy et al., 2019).

Our study has several limitations. One limitation of the current work is that we tested only a small collection $(n=16)$ of Mab strains in our RFB-CLR drug-drug potency interaction analysis. However, the conclusion that RFB potentiates CLR in an erm41 dependent manner is supported by previous studies, which showed similar trends [18,19]. A second limitation is, that we did not confirm the proposed mechanism by generating and analyzing targeted knock-outs in whiB7 and erm41. Finally, all the analyses were carried out in vitro and the effects need to be demonstrated in an animal model of Mab infection.

\section{Conclusions}

We have shown that co-treatment of erm41 positive Mab with RFB and CLR in vitro suppresses induction of CLR resistance. This effect appears to be due to inhibition of CLR mediated induction of the whiB7-erm41 system. This suggest that RFB-CLR treatment may be beneficial for patients infected with erm41 positive $M a b$.

\section{Materials and Methods}

Compounds. Clarithromycin was from Sigma-Aldrich and dissolved in acetone. Rifabutin was from SelleckChem and dissolved in 90\% dimethyl sulfoxide (DMSO).

Bacterial strains and culture media. For the checkerboard titration assay, both clinical isolates as well as Mycobacterium abscessus reference strains were used. The reference strains were obtained from the American Type Culture Collection (ATCC) and the Culture Collection University of Goteborg (CCUG) and comprised Mycobacterium abscessus subsp. abscessus (ATCC 19977), Mycobacterium abscessus subsp. bolletii (CCUG 50184-T) and Mycobacterium abscessus subsp. massiliense (CCUG 48898-T). The clinical isolates were obtained from the strain collection of the clinical microbiology laboratory at the National University Hospital, Singapore. The clinical strains were characterized previously [16]. For the measurement of the RNA levels by quantitative reverse transcription PCR (qRT-PCR), M. abscessus subsp. abscessus (ATCC 19977) was used. Liquid cultures were grown in standard mycobacterium medium, Middlebrook 7H9 broth (BD Difco) supplemented with 0.5\% albumin, 0.2\% glucose, $0.085 \%$ sodium chloride, $0.0003 \%$ catalase, $0.2 \%$ glycerol and $0.05 \%$ Tween 80 .

Checkerboard titration assay. This assay was carried out in 96-well microtiter plates as previously described using optical density to assess growth [24]. Eight concentrations of CLR were tested. For the erm 41 negative strains, the concentration range was $0-1.25 \mu \mathrm{M}$. For the erm 41 positive strains, the concentration range was $0-12.5 \mu \mathrm{M}$. The CLR concentrations were tested against 11 concentrations of rifabutin from $0-25 \mu \mathrm{M}$. Concentrations used were two-fold serial dilutions from the highest concentration. The fractional inhibitory concentration index (FICI) was used to analyze the results from the checkerboard assay. FICI was calculated by using the concentrations at which at least $90 \%$ inhibition of the culture in the well as compared to the drug free culture was observed. It was computed as FICI = [(concentration of drug A in combination/concentration of drug A when used alone) + (concentration of drug B in combination/concentration of drug B when used alone) [25]. Synergy was defined as FICI $\leq 0.5$, indifference was defined as $0.5<\mathrm{FICI} \leq 4$, and antagonism was defined as FICI $>4$ [25].

RNA extraction, quantitative reverse transcription PCR (qRT-PCR) analysis. M. abscessus subsp. abscessus (ATCC 19977) mid-log phase cultures were diluted to $\mathrm{OD}_{600}=0.1$ and exposed to antibiotics for 30 minutes. Cells were concentrated to $\mathrm{OD}_{600}=0.5$ and then lysed with Trizol (Ambion) and a mechanical disruption step using lysing matrix B in the FastPrep-24 5G (MP Biomedical). RNA was extracted using the PureLink RNA Mini Kit (Ambion), with on-column DNase (Ambion) treatment. The amount of RNA used was $50 \mathrm{ng} / \mu \mathrm{L}$ for all conditions. Random primers (Promega) and Superscript III reverse transcriptase (Invitrogen) were used for cDNA synthesis in the presence of RNase OUT (Invitrogen). Conditions for cDNA synthesis were: $50^{\circ} \mathrm{C}$ for $30 \mathrm{~min}$, followed by $70{ }^{\circ} \mathrm{C}$ for $15 \mathrm{~min}$. cDNA was then subjected to qPCR using FastStart Essential DNA Green Master (Roche) along with previously 
described primers; whiB7 (5'-CCTGTGGTTCGCGGAAA-3'/5'-CCCTGCTCAAGAATCTCACC-3') and erm41 (5'-CGAGCCCGCCCTACCAAGTCAC-3'/5'-CCGGCCCGTAGCGTCCAATG-3') [6,7]. Results were normalized to $r p s A$ using the previously described primer set; $r p s A$ (5'CAAGTAGCCGTCAACGACA-3'/5'-GACACCTTCGGTCTTGTAAC-3') [6]. A LightCycler 96 (Roche) was used for qPCR. Cycling conditions were: $95^{\circ} \mathrm{C}$ for $10 \mathrm{~min}$, followed by 45 cycles of $95{ }^{\circ} \mathrm{C}$ for $10 \mathrm{~s}$, $60{ }^{\circ} \mathrm{C}$ for $10 \mathrm{~s}$ and $72{ }^{\circ} \mathrm{C}$ for $10 \mathrm{~s}$. Results are presented as expression fold change of treated sample compared to untreated.

Supplementary Materials: The following are available online at http:/www.mdpi.com/2079-6382/9/2/72/s1, Table S1: Detailed $\mathrm{MIC}_{90}$ values (in $\mu \mathrm{M}$ ) of checkerboard assay results for the combination of clarithromycin and rifabutin against three reference strains and a collection of clinical isolates of M. abscessus.

Author Contributions: Conceptualization, D.B.A., M.L.G. and T.D.; Investigation, D.B.A.; writing一original draft preparation, D.B.A.; writing-review and editing, M.L.G. and T.D.; visualization, D.B.A.; supervision, M.L.G. and T.D.; funding acquisition, T.D. All authors have read and agreed to the published version of the manuscript.

Funding: This research was supported by the National Institute of Allergy and Infectious Diseases of the National Institutes of Health under award numbers R01AI132374 and U19AI142731. The content presented here is solely the responsibility of the authors and does not necessarily represent the official views of the National Institutes of Health.

Acknowledgments: We thank Wei Chang Huang, Taichung Veterans General Hospital, Taichung, Taiwan for the M. abscessus Bamboo strain and Jeanette Teo, National University Hospital, Singapore, for the clinical isolates. TD holds a Toh Chin Chye Visiting Professorship at the Department of Microbiology and Immunology, Yong Loo Lin School of Medicine, National University of Singapore. We thank Uday Ganapathy and Wassihun Aragaw, Center for Discovery and Innovation, Hackensack Meridian Health, Nutley, New Jersey, for discussion.

Conflicts of Interest: The authors declare no conflict of interest. The funders had no role in the design of the study; in the collection, analyses, or interpretation of data; in the writing of the manuscript, or in the decision to publish the results.

\section{References}

1. Griffith, D.E.; Aksamit, T.; Brown-Elliott, B.A.; Catanzaro, A.; Daley, C.; Gordin, F.; Holland, S.M.; Horsburgh, R.; Huitt, G.; Iademarco, M.F.; et al. An official ATS/IDSA statement: Diagnosis, treatment, and prevention of nontuberculous mycobacterial diseases. Am. J. Respir. Crit. Care Med. 2007, 175, 367-416. [CrossRef]

2. Haworth, C.S.; Banks, J.; Capstick, T.; Fisher, A.J.; Gorsuch, T.; Laurenson, I.F.; Leitch, A.; Loebinger, M.R.; Milburn, H.J.; Nightingale, M.; et al. British Thoracic Society guidelines for the management of non-tuberculous mycobacterial pulmonary disease (NTM-PD). Thorax 2017, 72, ii1-ii64. [CrossRef]

3. Lopeman, R.C.; Harrison, J.; Desai, M.; Cox, J.A.G. Mycobacterium abscessus: Environmental Bacterium Turned Clinical Nightmare. Microorganisms 2019, 7, 90. [CrossRef]

4. Koh, W.J.; Jeong, B.H.; Kim, S.Y.; Jeon, K.; Park, K.U.; Jhun, B.W.; Lee, H.; Park, H.Y.; Kim, D.H.; Huh, H.J.; et al. Mycobacterial Characteristics and Treatment Outcomes in Mycobacterium abscessus Lung Disease. Clin. Infect. Dis. 2017, 64, 309-316. [CrossRef]

5. Chen, J.; Zhao, L.; Mao, Y.; Ye, M.; Guo, Q.; Zhang, Y.; Xu, L.; Zhang, Z.; Li, B.; Chu, H. Clinical Efficacy and Adverse Effects of Antibiotics Used to Treat Mycobacterium abscessus Pulmonary Disease. Front. Microbiol. 2019, 10, 1977. [CrossRef]

6. Nash, K.A.; Brown-Elliott, B.A.; Wallace, R.J., Jr. A novel gene, erm(41), confers inducible macrolide resistance to clinical isolates of Mycobacterium abscessus but is absent from Mycobacterium chelonae. Antimicrob. Agents Chemother. 2009, 53, 1367-1376. [CrossRef]

7. Hurst-Hess, K.; Rudra, P.; Ghosh, P. Mycobacterium abscessus WhiB7 Regulates a Species-Specific Repertoire of Genes To Confer Extreme Antibiotic Resistance. Antimicrob. Agents Chemother. 2017, 61. [CrossRef]

8. Pryjma, M.; Burian, J.; Kuchinski, K.; Thompson, C.J. Antagonism between Front-Line Antibiotics Clarithromycin and Amikacin in the Treatment of Mycobacterium abscessus Infections is Mediated by the whiB7 Gene. Antimicrob. Agents Chemother. 2017, 61. [CrossRef]

9. Richard, M.; Gutierrez, A.V.; Kremer, L. Dissecting erm(41)-mediated macrolide inducible resistance in Mycobacterium abscessus. Antimicrob. Agents Chemother. 2019. [CrossRef] 
10. Tortoli, E.; Kohl, T.A.; Brown-Elliott, B.A.; Trovato, A.; Leao, S.C.; Garcia, M.J.; Vasireddy, S.; Turenne, C.Y.; Griffith, D.E.; Philley, J.V.; et al. Emended description of Mycobacterium abscessus, Mycobacterium abscessus subsp. abscessus and Mycobacteriumabscessus subsp. bolletii and designation of Mycobacteriumabscessus subsp. massiliense comb. nov. Int. J. Syst. Evol. Microbiol. 2016, 66, 4471-4479. [CrossRef]

11. Bastian, S.; Veziris, N.; Roux, A.L.; Brossier, F.; Gaillard, J.L.; Jarlier, V.; Cambau, E. Assessment of clarithromycin susceptibility in strains belonging to the Mycobacterium abscessus group by erm(41) and $\mathrm{rrl}$ sequencing. Antimicrob. Agents Chemother. 2011, 55, 775-781. [CrossRef] [PubMed]

12. Maurer, F.P.; Castelberg, C.; Quiblier, C.; Bottger, E.C.; Somoskovi, A. Erm(41)-dependent inducible resistance to azithromycin and clarithromycin in clinical isolates of Mycobacterium abscessus. J. Antimicrob. Chemother. 2014, 69, 1559-1563. [CrossRef] [PubMed]

13. Lee, M.R.; Sheng, W.H.; Hung, C.C.; Yu, C.J.; Lee, L.N.; Hsueh, P.R. Mycobacterium abscessus Complex Infections in Humans. Emerg. Infect. Dis. 2015, 21, 1638-1646. [CrossRef] [PubMed]

14. Brown-Elliott, B.A.; Vasireddy, S.; Vasireddy, R.; Iakhiaeva, E.; Howard, S.T.; Nash, K.; Parodi, N.; Strong, A.; Gee, M.; Smith, T.; et al. Utility of sequencing the erm(41) gene in isolates of Mycobacterium abscessus subsp. abscessus with low and intermediate clarithromycin MICs. J. Clin. Microbiol. 2015, 53, 1211-1215. [CrossRef]

15. Wu, M.L.; Aziz, D.B.; Dartois, V.; Dick, T. NTM drug discovery: Status, gaps and the way forward. Drug Discov. Today 2018, 23, 1502-1519. [CrossRef]

16. Aziz, D.B.; Low, J.L.; Wu, M.L.; Gengenbacher, M.; Teo, J.W.P.; Dartois, V.; Dick, T. Rifabutin Is Active against Mycobacterium abscessus Complex. Antimicrob. Agents Chemother. 2017, 61. [CrossRef]

17. Dick, T.; Shin, S.J.; Koh, W.J.; Dartois, V.; Gengenbacher, M. Rifabutin is active against Mycobacterium abscessus in mice. Antimicrob. Agents Chemother. 2019. [CrossRef]

18. Pryjma, M.; Burian, J.; Thompson, C.J. Rifabutin Acts in Synergy and Is Bactericidal with Frontline Mycobacterium abscessus Antibiotics Clarithromycin and Tigecycline, Suggesting a Potent Treatment Combination. Antimicrob. Agents Chemother. 2018, 62. [CrossRef]

19. Cheng, A.; Tsai, Y.T.; Chang, S.Y.; Sun, H.Y.; Wu, U.I.; Sheng, W.H.; Chen, Y.C.; Chang, S.C. In Vitro Synergism of Rifabutin with Clarithromycin, Imipenem, and Tigecycline against the Mycobacterium abscessus Complex. Antimicrob. Agents Chemother. 2019, 63. [CrossRef]

20. Ganapathy, U.S.; Dartois, V.; Dick, T. Repositioning rifamycins for Mycobacterium abscessus lung disease. Expert Opin. Drug Discov. 2019, 14, 867-878. [CrossRef]

21. Luthra, S.; Rominski, A.; Sander, P. The Role of Antibiotic-Target-Modifying and Antibiotic-Modifying Enzymes in Mycobacterium abscessus Drug Resistance. Front. Microbiol. 2018, 9, 2179. [CrossRef] [PubMed]

22. Schildkraut, J.A.; Pennings, L.J.; Ruth, M.M.; de Brouwer, A.P.; Wertheim, H.F.; Hoefsloot, W.; de Jong, A.; van Ingen, J. The differential effect of clarithromycin and azithromycin on induction of macrolide resistance in Mycobacterium abscessus. Future Microbiol. 2019, 14, 749-755. [CrossRef] [PubMed]

23. Choi, G.E.; Shin, S.J.; Won, C.J.; Min, K.N.; Oh, T.; Hahn, M.Y.; Lee, K.; Lee, S.H.; Daley, C.L.; Kim, S.; et al. Macrolide treatment for Mycobacterium abscessus and Mycobacterium massiliense infection and inducible resistance. Am. J. Respir. Crit. Care Med. 2012, 186, 917-925. [CrossRef] [PubMed]

24. Aziz, D.B.; Teo, J.W.P.; Dartois, V.; Dick, T. Teicoplanin - Tigecycline Combination Shows Synergy Against Mycobacterium abscessus. Front. Microbiol. 2018, 9, 932. [CrossRef]

25. Hsieh, M.H.; Yu, C.M.; Yu, V.L.; Chow, J.W. Synergy assessed by checkerboard. A critical analysis. Diagn. Microbiol. Infect. Dis. 1993, 16, 343-349. [CrossRef]

(C) 2020 by the authors. Licensee MDPI, Basel, Switzerland. This article is an open access article distributed under the terms and conditions of the Creative Commons Attribution (CC BY) license (http://creativecommons.org/licenses/by/4.0/). 